\title{
ACCURACY PREDICTIONS AND FINAL PROSPECTS FOR THE HIPPARCOS MISSION
}

\author{
L. LINDEGREN ${ }^{1}$ and J. KOVALEVSKY ${ }^{2}$ \\ ${ }^{1}$ Lund Observatory \\ Box 43, S-22100 Lund, Sweden \\ ${ }^{2}$ Observatoire de la Côte d'Azur \\ CERGA, Avenue Copernic, F-06130 Grasse, France
}

\begin{abstract}
The pre-launch accuracy prediction for the Hipparcos mission is reviewed with regard to the observed performance and expected lifetime of the satellite in its present orbit. Given the excellent quality of the data it is likely that the target accuracy of 2 milli-arcsec for typical programme stars can be achieved if operations continue at least until the end of 1993 . We emphasize that any extension of the operational lifetime beyond that point strongly enhances the scientific value of the results.
\end{abstract}

\section{Introduction}

Although the large-scale processing of Hipparcos data is still in an early stage, the two reduction consortia FAST and NDAC have accumulated considerable experience in dealing with the real satellite data: several weeks of data scattered throughout the first 15 months of the mission have been provisionally analysed, up to a first determination of positions and parallaxes, and in the FAST consortium the mass treatment of the complete data set from the beginning of the mission is well in progress. This is therefore a good opportunity to examine how the mission stands in terms of expected final accuracy. A few points may summarise the relevant experience so far:

- Compared with the nominal mission, valid observations are collected only during about $65 \%$ of the time (considerably less in the first few months of the mission).

- At the beginning of the mission the instrument performed better than nominal in terms of sensitivity. The geometrical stability is excellent.

- However, the sensitivity of the main detector chain is clearly deteriorating, with an $e$ folding time of 7 to 8 years.

- There is evidence for a gradual decrease in light modulation (MTF) that cannot be compensated by refocussing. The phenomenon may be caused by gradual deformation of the mirrors, but the reasons for this are not clear. The magnitude of the effect is almost $3 \%$ per year, corresponding to an $\epsilon$-folding time of about 35 years.

- From the viewpoint of consumables only (electrical power and nitrogen gas) a technical lifetime of almost five years appears feasible, i.e., until the fall of 1994.

In the following section we discuss how the instrument degradation will affect the astrometric precision. The pre-launch accuracy prediction is then partially revised to take into account the factors mentioned above. The main uncertainty is the mission lifetime. Results 
are therefore given for a range of assumed lifetimes from 2 to 5 years.

It should be recalled that the satellite data are first processed in batches of 3 to 8 hours representing the ensemble of valid observations collected during one orbital revolution of 10.7 hours. This produces the one-dimensional coordinates known as abscissae, measured on a reference great circle (RGC) defined independently for each orbit. The second step of the processing is the combination of the abscissae on all available RGC's into a consistent set of positions, proper motions and parallaxes for the programme stars. The precision currently obtained for the abscissae, and the accuracy expected after the final iteration, are the basic input to the revised prediction.

\section{Astrometric Effects Of Instrument Degradation}

The weight (inverse variance) of an individual observation, consisting of the phase determination of a photon-noise limited signal, is roughly proportional to the product of the instrument's sensitivity and MTF at the relevant spatial frequency. The total effect of the observed degradations is then roughly described by a function $\exp (-t / \tau)$ with $e$-folding time $\tau \simeq 6$ years. At the end of a hypothetical 5 -year mission the weights are thus only 40-45\% compared to the beginning of the mission. The effect on the astrometric results obtained after $P$ years can be assessed by analytical means. Assuming that measurements accumulate continuously and at a constant rate, the standard errors in the positions (at the weighted mean epoch) and parallaxes improve with $P$ according to:

$$
\sigma_{\lambda, \beta, \pi}^{2} \propto \frac{1}{\tau\left(1-e^{-P / \tau}\right)}=\frac{1}{P}\left[1+\frac{1}{2}\left(\frac{P}{\tau}\right)+\frac{1}{12}\left(\frac{P}{\tau}\right)^{2}+\cdots\right]
$$

The sky averages of the standard errors in longitude, latitude and parallax stand in a fixed proportion $\sigma_{\lambda} \cos \beta: \sigma_{\beta}: \sigma_{\pi}=1: 0.80: 1.26$ determined by the constant angle of $43^{\circ}$ between the spin axis and the sun. For the proper motions we find:

$$
\left(\frac{\sigma_{\mu \lambda}}{\sigma_{\lambda}}\right)^{2}=\left(\frac{\sigma_{\mu \beta}}{\sigma_{\beta}}\right)^{2}=\frac{1}{\tau^{2}} \frac{\left(1-e^{-P / \tau}\right)^{2}}{\left(1-e^{-P / \tau}\right)^{2}-\left(\frac{P}{\tau}\right)^{2} e^{-P / \tau}}=\frac{12}{P^{2}}\left[1+\frac{1}{20}\left(\frac{P}{\tau}\right)^{2}+\cdots\right]
$$

Thus, compared to a non-degrading instrument $(\tau=\infty)$ the standard errors of all astrometric results are increased by a factor $1+P /(4 \tau)+O(P / \tau)^{2}$. This factor, with $\tau=6$ years, is included in all the estimates reported below.

\section{A Revised Accuracy Prediction}

The accuracy prediction made before launch, as reported in [1], were based on extensive computer simulations of the instrument, the observations and the data reductions. These in turn were based on certain key assumptions on the available observing time per star, instrument sensitivity and MTF, perturbing forces, and so on. Practically all of these factors have to be modified in view of the observed performance in the current orbit. However, there is little point in repeating the simulations with revised assumptions, since the situation is in fact much more complex now and the outcome would anyway soon be 
superseded by 'real' results. Instead, we propose to sidestep the first (and most complex) part of the prediction by directly comparing the pre-launch computations, at the greatcircle (abscissa) level, with error estimates obtained in the reductions of real data. The second part of the prediction, which translates the great-circle accuracies into the expected astrometric accuracy, can be carried out with only minor modifications for the lifetime and data collection efficiency. We shall consider first a 'typical' star of magnitude 9 in the Hipparcos main detector photometric system $\left(H_{\mathrm{p}}\right)$. This is close to the median magnitude of the programme stars, which means that the standard errors quoted below should also be regarded as median values. Also, since the accuracy varies according to position on the sky, we consider a sky average of the standard errors. The expected variations with magnitude and latitude are given at the end of this section.

According to the pre-launch predictions the average standard error of the abscissa of a 9 th magnitude star would be 4.3 milli-arcsec (mas). This was based on an assumed 'geometrical' great-circle reduction (i.e., without taking full advantage of the smoothness of the satellite rotation) comprising some 1950 programme stars observed in a time interval of 10.7 hours. (By coincidence this is exactly one period in the current orbit; for brevity we now call this interval an 'orbit'.) In the real reductions we find an average formal standard error of 4.4 mas per orbit $\left(H_{\mathrm{p}}=9\right)$ using the geometrical solution method, but only for about 1550 stars on the average. The observational weight per unit time of the revised mission is thus about $75 \%$ of the predicted weight. That this number is greater than the data collection efficiency $(65 \%)$ is indicative of the very high performance of the instrument during its first year in orbit.

The performance at the great-circle level is discussed in another presentation at this meeting [2]. The estimated standard error in abscissa, using the smoothed great-circle solution method and after iterative improvement of the star catalogue and attitude determination, is about 3.6 mas per orbit $\left(H_{\mathrm{p}}=9\right)$. Assuming a (stochastic) data yield of $65 \%$ and the degradation factors of Section 2, this number translates into the following astrometric mean errors as function of mission length $P$ :

\begin{tabular}{llllll}
\hline$P$ & $\sigma_{\lambda} \cos \beta$ & $\sigma_{\beta}$ & $\sigma_{\pi}$ & $\sigma_{\mu \lambda} \cos \beta$ & $\sigma_{\mu \beta}$ \\
\hline $2.0 \mathrm{yr}$ & 1.6 mas & 1.3 mas & 2.1 mas & $2.9{\text { mas } \mathrm{yr}^{-1}}^{-1}$ & $2.3{\text { mas } \mathrm{yr}^{-1}}$ \\
2.5 & 1.5 & 1.2 & 1.9 & 2.1 & 1.7 \\
3.0 & 1.4 & 1.1 & 1.7 & 1.6 & 1.3 \\
4.0 & 1.2 & 1.0 & 1.6 & 1.1 & 0.9 \\
5.0 & 1.1 & 0.9 & 1.5 & 0.8 & 0.7 \\
\hline
\end{tabular}

The variation of the mean errors with magnitude can be assessed from the great-circle reductions. A plausible dependence is given by the following multiplicative factor:

\begin{tabular}{llllllllll}
\hline$H_{\mathrm{p}}$ & $\leq 5$ & 6 & 7 & 8 & 9 & 10 & 11 & 12 & 12.5 \\
\hline$\sigma_{H} / \sigma_{9}$ & 0.54 & 0.58 & 0.65 & 0.79 & 1.00 & 1.34 & 1.8 & 2.2 & 3 \\
\hline
\end{tabular}

As for the variations across the sky, the main systematic dependence is on ecliptic latitude $(\beta)$. For the longitude components of the positions and proper motions the correction factor varies from 1.35 at $\beta=0$ through a minimum of 0.65 around $\beta= \pm 45^{\circ}$, to 0.86 at 
$\beta= \pm 90^{\circ}$. The accuracy of the latitude components varies little with $\beta$. For the parallaxes the correction factor goes from 1.17 on the ecliptic to 0.71 at the poles.

\section{Discussion}

The prediction described above is basically a propagation of formal standard errors through a sequence of estimation procedures, starting with the photon noise of the detector signal and successively adding a number of other (known) error sources. It does not include an explicit 'safety' margin. By its very nature it will tend to give a somewhat optimistic picture of what can be achieved. It is therefore important to confront it with some more empirical accuracy estimate. One such possibility is offered by the first test computation of trigonometric parallaxes, using a small portion of the first 15 months of Hipparcos data [3]. From the distribution of negative parallaxes obtained in that solution, an external rms parallax error of 5.4 mas could be estimated for a subset of 3500 stars. Proper motions were not adjusted in that solution and so contribute statistically to the observed dispersion. Correcting for this gives an estimated standard error of 4.8 mas for the parallaxes. From the known geometry of the great-circle scans included in the solution this can be translated into an average abscissa accuracy of 4.7 mas per orbit. This is $30 \%$ higher than the value assumed in the previous section. Considering the unfavourable geometry of the provisional parallax solution and the probable contamination by double stars, we would regard these $30 \%$ as an upper limit to the degree of uncertainty in the accuracy prediction.

Adopting a conservative margin of $30 \%$ on the standard errors in Section 3 we conclude that the target accuracy of 2 mas should be reached after $P=4$ years (end of 1993). Again it should be noted that this is roughly a median value for the entire observing programme, and that the standard errors on individual (faint) stars may be several times greater.

The improvement with increasing $P$ is most apparent in the proper motions. This reflects also on the quality of the positional reference frame, which is limited by the proper motions as soon as the epoch difference exceeds a few years. However, it should also be remembered that the volume of space (and number of stars) for which trigonometric distances are obtained with a given relative error increases as $\sigma_{\pi}^{-3}$. The seemingly modest improvement in $\sigma_{\pi}$ beyond $P=3$ or 4 years actually represents a huge addition to what can be achieved in terms of astrophysical investigations. Thus, from a scientific viewpoint it is absolutely mandatory that this already successful satellite is operated as long as technically possible.

\section{References}

[1] Perryman, M.A.C., Lindegren, L., Murray, C.A., Høg, E. and Kovalevsky, J. (1989), The Hipparcos Mission, ESA SP-1111, Vol. III, European Space Agency, Noordwijk, Chapter 18.

[2] Kovalevsky, J., Petersen, C., van der Marel, H. and Donati, F. (1991), Performances of the Hipparcos data reduction on the great circle, this volume.

[3] Lindegren, L., van Leeuwen, F., Petersen, C., Perryman, M.A.C and Söderhjelm, S. (1991), Positions and parallaxes from the Hipparcos satellite: A first attempt at a global astrometric solution, Astronomy \& Astrophysics (in press). 